\title{
Magnetic moments of the spin-1/2 doubly charmed baryons in covariant baryon chiral perturbation theory
}

\author{
Ming-Zhu Liu, Yang Xiao, and Li-Sheng Geng* \\ School of Physics and Nuclear Energy Engineering and International Research Center for Nuclei \\ and Particles in the Cosmos and Beijing Key Laboratory of Advanced Nuclear Materials and Physics, \\ Beihang University, Beijing 100191, China
}

(Received 3 July 2018; published 31 July 2018)

\begin{abstract}
Inspired by the recent discovery of the $\Xi_{c c}^{++}$by the LHCb Collaboration, we study the magnetic moments of the spin-1/2 doubly charmed baryons up to the next-to-leading order in covariant baryon chiral perturbation theory with the extended-on-mass-shell renormalization scheme. There are three low energy constants at this order: $a_{1}, a_{2}$, and $g_{a}$. The latest lattice QCD simulations allow us to fix a combination of $a_{1}$ and $a_{2}$, while the axial-vector coupling $g_{a}$ can be determined in three different ways: by fitting to the lattice QCD data, by the quark model, or by the heavy antiquark diquark symmetry. The magnetic moments of the spin-1/2 doubly charmed baryons, $\Xi_{c c}^{d}$ and $\Xi_{c c}^{s}$, can then be predicted. We compare our results with those obtained in the heavy baryon chiral perturbation theory and other approaches, and point out some inconsistencies between the lattice QCD simulations and the quark model.
\end{abstract}

DOI: 10.1103/PhysRevD.98.014040

\section{INTRODUCTION}

The doubly charmed baryons, $\Xi_{c c}^{u}$, $\Xi_{c c}^{d}$, and $\Xi_{c c}^{s}$, are composed of two charm quarks and one light quark. One of them, $\Xi_{c c}^{+}$, with a mass of $3519 \pm 2 \mathrm{MeV}$ was first reported by the SELEX Collaboration [1,2]. Unfortunately, no other collaborations found such a state. Recently, the LHCb Collaboration observed another doubly charmed baryon state $\Xi_{c c}^{++}$with a mass of $3621.4 \pm 0.78 \mathrm{MeV}$, which has inspired many theoretical studies on its weak [3-5], strong, and radiative decays [6-8].

The magnetic moment of a hadron is one of its most important properties, which encodes crucial information on its inner structure. In the past, many phenomenological models have been used to study the magnetic moments of $\Xi_{c c}$ [9-17]. More recently, they have been calculated in heavy baryon chiral perturbation theory (HB ChPT) [18] and QCD sum rules [19]. In this work, we will study the magnetic moments of the spin- $1 / 2$ doubly charmed baryons up to the next-to-leading order (NLO) in covariant baryon chiral perturbation theory (BChPT) with the extended-on-massshell (EOMS) renormalization scheme. In the present work, we will contrast the ChPT results with the lattice QCD data of Ref. [20] to determine the unknown low energy constants

\footnotetext{
*lisheng.geng@buaa.edu.cn
}

Published by the American Physical Society under the terms of the Creative Commons Attribution 4.0 International license. Further distribution of this work must maintain attribution to the author(s) and the published article's title, journal citation, and DOI. Funded by SCOAP.
(LECs). In many recent studies (see, e.g., Refs. [21,22]), it has been shown that the EOMS BChPT can provide a better description of the lattice QCD quark-mass dependent results than its nonrelativistic counterpart.

Chiral perturbation theory (ChPT) is a low energy effective field theory of QCD, which plays an important role in our understanding of the nonperturbative strong interaction. In ChPT, relevant Feynman diagrams contributing to a certain process are organized as an expansion in powers of the external momenta and light quark masses. In the center of such an expansion is a power counting scheme, first proposed by Weinberg [23]. However, in the one-baryon sector, the naive power counting breaks down because of the large nonzero baryon mass $m_{0}$ in the chiral limit. To overcome this issue, HB ChPT, which performs a dual expansion in terms of both $1 / m_{0}$ and the chiral expansion, was proposed $[24,25]$. Later, two relativistic schemes were also proposed, i.e., the infrared (IR) [26] and EOMS [27] schemes. For a recent and concise summary of different schemes, see, e.g., Ref. [28].

The EOMS scheme has already been successfully applied to study many physical observables such as the magnetic moments [22,29-31], the masses, and sigma terms [21, 32-34] of the octet and decuplet baryons, the hyperon vector couplings [35,36], the axial vector charges [37], the pionnucleon scattering [38,39], the nucleon Compton scattering [40], the neutral pion photo production [41], the scattering of pseudoscalar mesons off $D / B$ mesons [42-44], the $D D^{*}$ scattering [45], and the $\Xi_{c c}$ masses and sigma terms $[46,47]$. It will be interesting to see how it describes the magnetic moments of the $\Xi_{c c}$ baryons, particularly from the perspective of lattice QCD simulations. 
This work is organized as follows. In Sec. II, we provide the theoretical ingredients and calculate the pertinent Feynman diagrams. Results and discussions are given in Sec. III, followed by a short summary in Sec. IV.

\section{THEORETICAL FORMALISM}

The magnetic moments of doubly charmed baryons are defined via the matrix elements of the electromagnetic current $J_{\mu}$ in the following way:

$$
\left\langle\Psi\left(p^{\prime}\right)\left|J_{\mu}\right| \Psi(p)\right\rangle=\bar{u}\left(p^{\prime}\right)\left[\gamma_{\mu} F_{1}^{B}(t)+\frac{i \sigma_{\mu \nu} q^{\nu}}{2 m_{B}} F_{2}^{B}(t)\right] u(p),
$$

where $\bar{u}\left(p^{\prime}\right)$ and $u(p)$ are Dirac spinors, $m_{B}$ is the chiral limit doubly charmed baryon mass, and $F_{1}^{B}(t)$ and $F_{2}^{B}(t)$ denote the Dirac and Pauli form factors, respectively. The four-momentum transfer is defined as $q=p^{\prime}-p$ and $t=q^{2}$. At $t=0, F_{2}^{B}(0)$ is the so-called anomalous magnetic moment, $\kappa_{B}$, and the magnetic moment is $\mu_{B}=\kappa_{B}+Q_{B}$, where $Q_{B}$ is the charge of the doubly charmed baryon. Up to NLO, there are three Feynman diagrams contributing to the magnetic moments of the $\Xi_{c c}$ as shown in Fig. 1, where diagram (a) is of $\mathcal{O}\left(p^{2}\right)$ and diagrams (b) and (c) are of $\mathcal{O}\left(p^{3}\right)$.

\section{A. Tree level diagram}

The leading order (tree-level) contribution is provided by the following Lagrangian:

$\mathcal{L}_{M B}^{(2)}=a_{1} \frac{1}{8 m_{B}} \bar{H} \sigma^{\mu \nu} \hat{F}_{\mu \nu}^{+} H+a_{2} \frac{1}{8 m_{B}} \bar{H} \sigma^{\mu \nu} H \operatorname{Tr}\left(F_{\mu \nu}^{+}\right)$,

where $\sigma^{\mu \nu}=\frac{i}{2}\left[\gamma^{\mu}, \gamma^{\nu}\right], F_{\mu \nu}^{+}=|e|\left(u^{\dagger} Q^{\prime} F_{\mu \nu} u+u Q^{\prime} F_{\mu \nu} u^{\dagger}\right)$, $F_{\mu \nu}=\partial_{\mu} A_{\nu}-\partial_{\nu} A_{\mu}, \quad \hat{F}_{\mu \nu}^{+}=F_{\mu \nu}^{+}-\frac{1}{3} \operatorname{Tr}\left(F_{\mu \nu}^{+}\right), \quad$ and $Q^{\prime}=$ $\operatorname{diag}(2,1,1)$ is the baryon charge matrix, $u=\exp \left[i \Phi / 2 f_{\phi}\right]$ with $\Phi$ the unimodular matrix containing the pseudoscalar nonet, and $f_{\phi}$ the pseudoscalar decay constant. In the numerical analysis, we use the following physical values

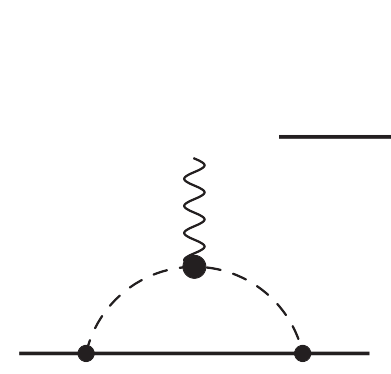

(b)

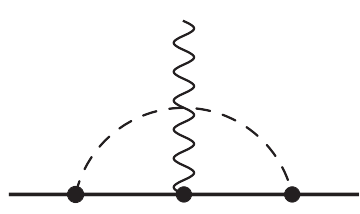

(c)
FIG. 1. Feynman diagrams contributing to the magnetic moments of the $\Xi_{c c}$ baryons: (a) tree level, (b) meson pole, and (c) baryon pole. The solid lines denote the doubly charmed baryons, the dashed lines denote the Nambu-Goldstone bosons, and the wiggly lines indicate the photon. The heavy dots indicate $\mathcal{O}\left(p^{2}\right)$ vertices, and the normal dots denote $\mathcal{O}(p)$ vertices.
TABLE I. $\mathcal{O}\left(p^{2}\right)$ coefficients appearing in Eq. (3).

\begin{tabular}{cccc}
\hline \hline & $\Xi_{c c}^{u}$ & $\Xi_{c c}^{d}$ & $\Xi_{c c}^{s}$ \\
\hline$\alpha_{B}$ & $2 / 3$ & $-1 / 3$ & $-1 / 3$ \\
$\beta_{B}$ & 4 & 4 & 4 \\
\hline \hline
\end{tabular}

for the decay constants: $f_{\pi}=92.4 \mathrm{MeV}, f_{K}=1.22 f_{\pi}$, $f_{\eta}=1.3 f_{\pi}$. For $m_{B}$, we use the SU(3) average of the lattice QCD results, i.e., $m_{B}=3722 \mathrm{MeV}$ [20]. The $\Xi_{c c}$ baryons are contained in a column $H$, which reads

$$
H=\left(\begin{array}{l}
\Xi_{c c}^{u} \\
\Xi_{c c}^{d} \\
\Xi_{c c}^{s}
\end{array}\right) .
$$

The tree level contributions to the magnetic moments can be easily obtained as

$$
\mu_{B}^{(2)}=\alpha_{B} a_{1}+\beta_{B} a_{2},
$$

where $\alpha_{B}=\left(\left\langle\bar{H} Q^{\prime} H\right\rangle-\frac{1}{3} \bar{H} H\left\langle Q^{\prime}\right\rangle\right)$ and $\beta_{B}=\bar{H} H\left\langle Q^{\prime}\right\rangle$ are given in Table I. We will determine the two LECs $a_{1}$ and $a_{2}$ by fitting to the lattice QCD simulations.

\section{B. Loop diagrams}

At $\mathcal{O}\left(p^{3}\right)$, there are two Feynman diagrams, the so-called baryon-pole and meson-pole diagrams, as shown in Fig. 1.

The Lagrangian for a doubly charmed baryon interacting with a Nambu-Goldstone boson (NGB) is

$$
\mathcal{L}_{M B B}^{(1)}=\frac{g_{a}}{2} \bar{H} \gamma^{\mu} \gamma^{5} u_{\mu} H,
$$

where $u_{\mu}=\left[u^{\dagger}\left(\partial_{\mu}-i r_{\mu}\right) u-u\left(\partial_{\mu}-i l_{\mu}\right) u^{\dagger}\right]$, and $g_{a}$ is the axial-vector coupling constant.

The Lagrangian describing the interaction between a baryon and a photon is of $\mathcal{O}(p)$ and reads

$$
\mathcal{L}_{B}^{(1)}=i \bar{H} \gamma^{u} D_{\mu} H,
$$

where $D_{\mu}=\partial_{\mu}+\Gamma_{\mu}, \Gamma_{\mu}=\frac{1}{2}\left[u^{\dagger}\left(\partial_{\mu}-i r_{\mu}\right) u+u\left(\partial_{\mu}-i l_{\mu}\right) u^{\dagger}\right]=$ $\frac{1}{2}\left(u^{\dagger} \partial_{\mu} u+u \partial_{\mu} u^{\dagger}\right)-\frac{i}{2}\left(u^{\dagger} r_{\mu} u+u l_{\mu} u^{\dagger}\right)=-i e Q^{\prime} A_{\mu}$.

The Lagrangian describing the interaction between a meson and a photon is of $\mathcal{O}\left(p^{2}\right)$ and reads

$$
\mathcal{L}_{M}^{(2)}=\frac{f_{\phi}^{2}}{4} \operatorname{Tr}\left[\nabla_{\mu} U\left(\nabla^{\mu} U\right)^{\dagger}\right],
$$

where $\nabla_{\mu} U=\partial_{\mu} U+i e A_{\mu}(Q U-U Q)$ and $Q=$ $\operatorname{diag}(2 / 3,-1 / 3,-1 / 3)$.

From these, one can easily obtain the loop contributions to the magnetic moments, i.e.,

$$
\mu_{\text {loop }}^{i}=c_{b}^{i}(\phi) H^{b}\left(m_{\phi}\right)+c_{m}^{i}(\phi) H^{m}\left(m_{\phi}\right),
$$

where $c_{b}^{i}(\phi)$ and $c_{m}^{i}(\phi)$ are tabulated in Tables II and III, $i$ runs over $\Xi_{c c}^{u}, \Xi_{c c}^{d}$, and $\Xi_{c c}^{s}$, and $\phi$ denotes $\pi, K$, or $\eta$. The 
TABLE II. Coefficients of the baryon-pole contributions appearing in Eq. (7).

\begin{tabular}{lccc}
\hline \hline$c_{b}$ & $\Xi_{c c}^{u}$ & $\Xi_{c c}^{d}$ & $\Xi_{c c}^{s}$ \\
\hline$\pi$ & 4 & 5 & 0 \\
$\eta$ & $2 / 3$ & $1 / 3$ & $4 / 3$ \\
$K$ & 2 & 2 & 6 \\
\hline \hline
\end{tabular}

TABLE III. Coefficients of the meson-pole contributions appearing in Eq. (7).

\begin{tabular}{lccc}
\hline \hline$c_{m}$ & $\Xi_{c c}^{u}$ & $\Xi_{c c}^{d}$ & $\Xi_{c c}^{s}$ \\
\hline$\pi$ & -2 & 2 & 0 \\
$K$ & -2 & 0 & 2 \\
\hline \hline
\end{tabular}

loop functions $H^{b}\left(m_{\phi}\right)$ and $H^{m}\left(m_{\phi}\right)$ with $m_{\phi}$ the mass of a NGB are

$$
\begin{aligned}
H^{b}\left(m_{\phi}\right)= & -\frac{g_{a}^{2}}{16 \pi^{2} f_{\phi}^{2}}\left[m_{B}^{2}+2 m_{\phi}^{2}+\frac{m_{\phi}^{2}}{m_{B}^{2}}\left(m_{B}^{2}-m_{\phi}^{2}\right) \log \left(\frac{m_{\phi}^{2}}{m_{B}^{2}}\right)\right. \\
& \left.+\frac{2 m_{\phi}^{3}\left(m_{\phi}^{2}-3 m_{B}^{2}\right)}{m_{B}^{2} \sqrt{4 m_{B}^{2}-m_{\phi}^{2}}} \arccos \left(\frac{m_{\phi}}{2 m_{B}}\right)\right], \\
H^{m}\left(m_{\phi}\right)= & \frac{g_{a}^{2}}{16 \pi^{2} f_{\phi}^{2}}\left[-m_{B}^{2}+2 m_{\phi}^{2}+\frac{m_{\phi}^{2}}{m_{B}^{2}}\left(2 m_{B}^{2}-m_{\phi}^{2}\right) \log \left(\frac{m_{\phi}^{2}}{m_{B}^{2}}\right)\right. \\
& \left.+\frac{2 m_{\phi}\left(m_{\phi}^{4}-4 m_{\phi}^{2} m_{B}^{2}+2 m_{B}^{2}\right)}{m_{B}^{2} \sqrt{4 m_{B}^{2}-m_{\phi}^{2}}} \arccos \left(\frac{m_{\phi}}{2 m_{B}}\right)\right] .
\end{aligned}
$$

Up to NLO, the total magnetic moments are a sum of the tree and loop contributions, and they are usually expressed in units of the nucleon magneton $\mu_{N}$. In the end, we obtain

$$
\begin{aligned}
\mu_{\Xi_{c c}^{u}}= & \frac{m_{N}}{m_{B}}\left(2+\frac{2}{3} a_{1}+4 a_{2}+c_{b}^{1}(\phi) H^{b}\left(m_{\phi}\right)\right. \\
& \left.+c_{m}^{1}(\phi) H^{m}\left(m_{\phi}\right)\right), \\
\mu_{\Xi_{c c}^{d}}= & \frac{m_{N}}{m_{B}}\left(1-\frac{1}{3} a_{1}+4 a_{2}+c_{b}^{2}(\phi) H^{b}\left(m_{\phi}\right)\right. \\
& \left.+c_{m}^{2}(\phi) H^{m}\left(m_{\phi}\right)\right), \\
\mu_{\Xi_{c c}^{s}}= & \frac{m_{N}}{m_{B}}\left(1-\frac{1}{3} a_{1}+4 a_{2}+c_{b}^{3}(\phi) H^{b}\left(m_{\phi}\right)\right. \\
& \left.+c_{m}^{3}(\phi) H^{m}\left(m_{\phi}\right)\right),
\end{aligned}
$$

where $m_{N}=940 \mathrm{MeV}$ is the nucleon mass.
TABLE IV. Lattice QCD magnetic moments and masses of $\Xi_{c c}^{d}$ and $\Xi_{c c}^{s}$ at different $m_{\pi}^{2}$ [20].

\begin{tabular}{cccccc}
\hline \hline & $m_{\pi}^{2}$ & $m_{\Xi_{c c}^{d}}$ & $m_{\Xi_{c c}^{s}}$ & $\mu_{\Xi_{c c}^{d}}$ & $\mu_{\Xi_{c c}^{s}}$ \\
\hline Lattice & 0.490 & $3.810(12)$ & $3.861(17)$ & $0.412(13)$ & $0.389(18)$ \\
& 0.325 & $3.740(13)$ & $3.806(12)$ & $0.404(12)$ & $0.386(11)$ \\
& 0.168 & $3.708(16)$ & $3.788(16)$ & $0.410(20)$ & $0.400(11)$ \\
& 0.090 & $3.689(18)$ & $3.781(28)$ & $0.416(19)$ & $0.402(15)$ \\
\hline \hline
\end{tabular}

\section{RESULTS AND DISCUSSIONS}

In the following, we determine the LECs $a_{1}$ and $a_{2}$ by fitting to the lattice QCD simulations of Ref. [20], which are given in Table IV. The LEC $g_{a}$ will be determined by three ways: (case 1) by fitting to the lattice QCD simulations, (case 2) by the heavy antiquark diquark symmetry (HADS), or (case 3 ) by the quark model. To quantify the agreement with the lattice QCD data, we use the $\chi^{2}$ defined as

$$
\chi_{j}^{2}=\sum_{k=1}^{4} \frac{\left(\mu_{\mathrm{theo}}^{k}-\mu_{l \mathrm{QCD}}^{k}\right)^{2}}{d_{k}^{2}}
$$

where $\mu_{\text {theo }}^{k}$ and $\mu_{l \mathrm{QCD}}^{k}\left(d_{k}\right)$ are the magnetic moments (uncertainties) obtained in BChPT and those of the lattice QCD simulations of Table IV for $\Xi_{c c}^{d}(j=1)$ and $\Xi_{c c}^{s}$ $(j=2)$, respectively.

From Eq. (10), it is clear that since the lattice QCD data are only available for $\Xi_{c c}^{d}$ and $\Xi_{c c}^{s}$, we cannot determine the LECs $a_{1}$ and $a_{2}$ simultaneously. Only the combination $c_{1}=-\frac{1}{3} a_{1}+4 a_{2}$ can be fixed. As a result, we cannot predict the magnetic moment of $\Xi_{c c}^{u}$ without further inputs.

\section{A. Results at $\mathcal{O}\left(p^{2}\right)$}

If we just consider the tree level contribution, we have only one LEC, $c_{1}$. It can be determined by fitting to the lattice QCD data. The resulting value and $\chi^{2}$ are shown in Table V. The predicted magnetic moments of $\Xi_{c c}$ at the physical pion mass are

$$
\mu_{\Xi_{c c}^{d}}=\mu_{\Xi_{c c}^{s}}=\frac{m_{N}}{m_{B}}\left(c_{1}+1\right)=0.401(3) \mu_{N},
$$

where the number in the parenthesis is the uncertainty at the $68 \%$ confidence level.

TABLE V. $\mathcal{O}\left(p^{2}\right)$ LEC determined by fitting to the lattice QCD data of Table IV [20] and the corresponding $\chi^{2}$.

\begin{tabular}{cccc}
\hline \hline $\mathcal{O}\left(p^{2}\right)$ & $c_{1}$ & $\chi_{\Xi_{c c}^{d}}$ & $\chi_{\Xi_{c c}^{s}}$ \\
\hline & $0.586(19)$ & 1.678 & 2.238 \\
\hline
\end{tabular}


TABLE VI. Low energy constants $c_{1}$ and $g_{a}$ and the corresponding $\chi^{2}$ of each case described in the text.

\begin{tabular}{|c|c|c|c|c|c|c|}
\hline & \multicolumn{2}{|c|}{ Case 1} & \multicolumn{2}{|c|}{ Case 2} & \multicolumn{2}{|c|}{ Case 3} \\
\hline & EOMS & $\mathrm{HB}$ & EOMS & $\mathrm{HB}$ & EOMS & $\mathrm{HB}$ \\
\hline$c_{1}$ & $0.535(82)$ & $0.542(70)$ & 0.249 (19) & $0.264(19)$ & $0.060(19)$ & $0.083(21)$ \\
\hline$g_{a}$ & $0.078(61)$ & $0.074(56)$ & 0.2 & 0.2 & 0.25 & 0.25 \\
\hline$\chi_{\Xi_{c c}^{d}}^{2}$ & 1.494 & 1.513 & 11.175 & 13.180 & 27.797 & 32.785 \\
\hline$\chi_{\Xi_{c c}^{s}}^{2}$ & 2.039 & 2.048 & 4.268 & 4.448 & 8.664 & 9.155 \\
\hline
\end{tabular}

\section{B. Results at $\mathcal{O}\left(p^{3}\right)$}

At $\mathcal{O}\left(p^{3}\right)$, the meson masses will contribute via the loop diagrams. We determine the eta and kaon masses by the leading order ChPT. Setting the strange quark mass to its physical value, we obtain the following relation:

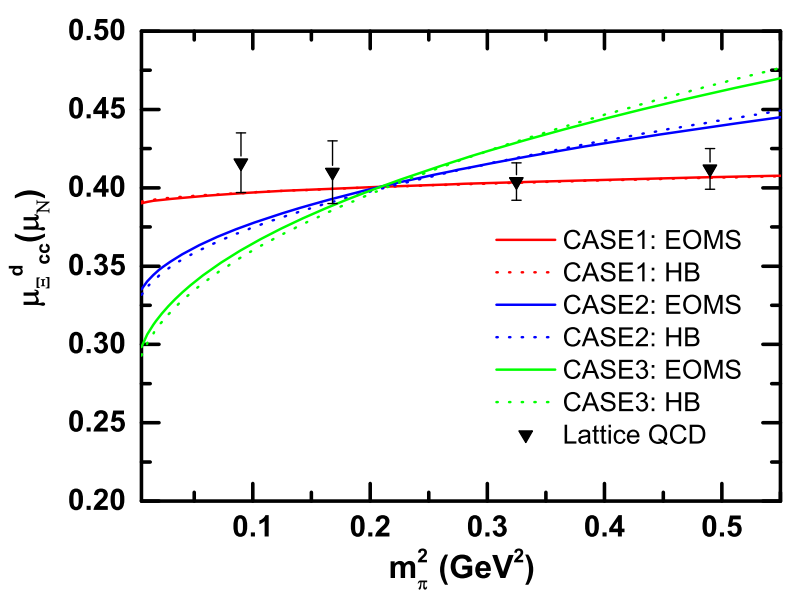

FIG. 2. Magnetic moment of $\Xi_{c c}^{d}$ as a function of $m_{\pi}^{2}$. The theoretical results are obtained with the LEC $c_{1}$ determined by fitting to the lattice QCD data and the LEC $g_{a}$ determined in three different ways as explained in the text.

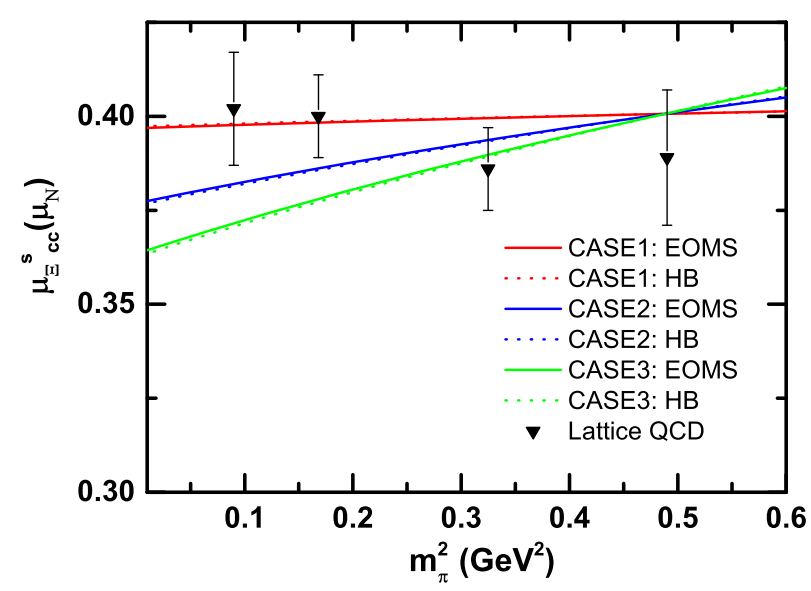

FIG. 3. Same as Fig. 2, but for the magnetic moment of $\Xi_{c c}^{s}$.

$$
\begin{aligned}
& m_{K}^{2}=\frac{1}{2} m_{\pi}^{2}+\left(m_{K}^{2}-\frac{1}{2} m_{\pi}^{2}\right)_{\mathrm{phys}}, \\
& m_{\eta}^{2}=\frac{1}{3} m_{\pi}^{2}+\frac{4}{3}\left(m_{K}^{2}-\frac{1}{2} m_{\pi}^{2}\right)_{\mathrm{phys}} .
\end{aligned}
$$

Fitting to the lattice QCD simulations tabulated in Table IV and with the LEC $g_{a}$ determined in the three different ways explained above, the resulting LECs as well as the $\chi^{2}$ are tabulated in Table VI. For the sake of comparison, we show the results obtained in HBChPT as well. It is seen that the lattice QCD data seem to prefer a $g_{a}$ that is smaller than that predicted either by the quark model or the HADS. Furthermore, as $g_{a}$ becomes larger, the EOMS BChPT description of the lattice QCD data becomes slightly better than that of the HBChPT, although for case 1 , where $g_{a}$ is taken as a free LEC, the descriptions are of similar quality.

In Figs. 2 and 3, we plot the predicted magnetic moments of $\Xi_{c c}^{d}$ and $\Xi_{c c}^{s}$ as a function of $m_{\pi}^{2}$, in comparison with the lattice QCD data. As can be clearly seen, there is not much difference between the EOMS and HB results. However, somewhat surprisingly, using the $g_{a}$ determined by either the quark model or the HADS yields unacceptable fits. This indicates that there is a considerable discrepancy between the quark model (the HADS) and the lattice QCD simulations of Ref. [20]. ${ }^{1}$

Note that we have used all of the eight sets of lattice QCD data and some of them are obtained with pion masses as large as $700 \mathrm{MeV}$. They are probably beyond the limit where an $\mathcal{O}\left(p^{3}\right)$ BChPT study can be trusted. Nevertheless, it is clear from the plots that limiting ourselves to the lattice QCD data with smaller pion masses will not change qualitatively any of our conclusions.

In contrary to the nucleon case where the HB and EOMS results can differ substantially [22], for the doubly charmed $\Xi_{c c}$ baryons, the loop contributions are much suppressed. This can be easily seen from the small $g_{a} \approx 0.08-0.25$, which is less than a fifth of the axial-vector coupling of the nucleon, $g_{A}=1.26$. As shown in Fig. 4, the magnetic

\footnotetext{
${ }^{1}$ One may need go to the next-to-next-to-leading order (NNLO) to draw a firm conclusion. However, at present, this is not feasible because of the increase in the number of free LECs in BChPT and the limited lattice QCD data.
} 


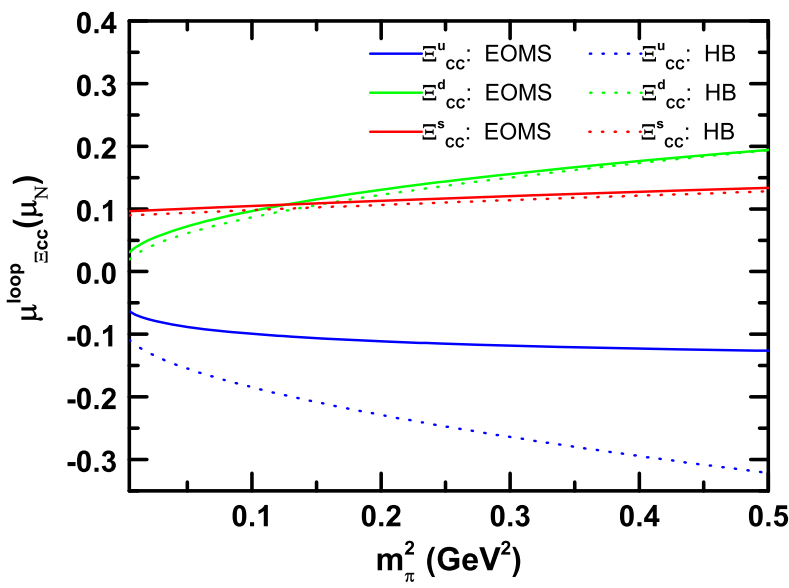

FIG. 4. Loop contributions to the magnetic moments of $\Xi_{c c}$ as a function of $m_{\pi}^{2}$ for $g_{a}=0.25$.

moments of $\Xi_{c c}^{d}$ and $\Xi_{c c}^{s}$ receive only small relativistic corrections, while for $\Xi_{c c}^{u}$ the correction is slightly larger. This can serve a nontrivial test of the ChPT results once more refined lattice QCD data become available.

One should note that the fits to the lattice QCD simulations are only of an exploratory nature. In the present work, we have not taken into account finite volume corrections and continuum extrapolations. In addition, because of the limited lattice QCD data, we have not performed a full study of truncation errors, different from the study of the magnetic moments of the ground-state octet baryons [22].

In Table VII, we compare the predicted magnetic moments of $\Xi_{c c}$ (case 1) with those obtained in other approaches. One finds that the theoretical results are very much scattered. Clearly, more investigations are needed to understand the current situation. Such studies may provide vital information on the nature of these $\Xi_{c c}$ baryons.

A few comments are in order. Clearly, the lattice QCD results of Ref. [20] and the present BChPT results (based on the same lattice QCD data) are not consistent with the quark model results. This is somehow surprising because one naively expects that the quark model becomes a better approximation of QCD with increasing quark masses as realized in lattice QCD simulations. In addition, the rather weak pion mass dependence of the lattice QCD data dictates a $g_{a}$ much smaller than the one predicted by either the quark model or the HADS. This may also be seen as a sign of the inconsistency between the quark model and the lattice QCD simulations. It remains an interesting issue to understand such discrepancies.
TABLE VII. Comparison of the magnetic moments of $\Xi_{c c}$ with those predicted by other approaches. Note that the EOMS BChPT results are obtained by fitting to the lattice QCD data of Ref. [20] up to NLO taking $c_{1}$ and $g_{a}$ as free LECs.

\begin{tabular}{|c|c|c|c|}
\hline$\Psi$ & $\Xi_{c c}^{u}\left(\mu_{N}\right)$ & $\Xi_{c c}^{d}\left(\mu_{N}\right)$ & $\Xi_{c c}^{s}\left(\mu_{N}\right)$ \\
\hline QCD sum rule [19] & 0.84 & 0.46 & 0.43 \\
\hline HBChPT [18] & -0.25 & 0.85 & 0.78 \\
\hline Lattice QCD [20] & $\cdots$ & 0.425 & 0.413 \\
\hline QM [9] & -0.12 & 0.80 & 0.69 \\
\hline RQM [10] & -0.10 & 0.86 & 0.72 \\
\hline Skyrmion [11] & -0.47 & 0.98 & 0.59 \\
\hline NQM [12] & -0.20 & 0.79 & 0.64 \\
\hline$\chi \mathrm{CQM}[13]$ & 0.006 & 0.84 & 0.70 \\
\hline RTQM [14] & 0.13 & 0.72 & 0.67 \\
\hline NRQM [15] & -0.20 & 0.78 & 0.63 \\
\hline MIT bag model [16] & 0.17 & 0.86 & 0.84 \\
\hline CLP [17] & -0.154 & 0.778 & 0.657 \\
\hline EOMS BChPT* & $\cdots$ & $0.392(13)$ & $0.397(15)$ \\
\hline
\end{tabular}

\section{SUMMARY}

We calculated the magnetic moments of the $\Xi_{c c}$ baryons in covariant baryon chiral perturbation theory with the extended-on-mass-shell scheme up to the next-to-leading order. The relevant low-energy constants are determined by fitting to lattice QCD simulations. We showed that the lattice QCD data support an axial-vector coupling $g_{a}$ smaller than those predicted by either the quark model or the heavy antiquark diquark symmetry. In addition, we found that relativistic corrections are very small for $\Xi_{c c}^{d}$ and $\Xi_{c c}^{s}$, but relatively large for $\Xi_{c c}^{u}$. This should be tested by future lattice QCD simulations. On the other hand, we notice that the present lattice QCD results are inconsistent with those of the quark model. More studies, particularly lattice QCD studies, are therefore in urgent need given the remarkable experimental progress achieved in the last few years.

\section{ACKNOWLEDGMENTS}

This work is partly supported by the National Natural Science Foundation of China under Grants No. 11522539, No. 11735003 and the fundamental Research Funds for the Central Universities.

Note added.-Recently, a study of the electromagnetic form factors of the $\Xi c c$ baryons in the same theoretical framework appeared in arXiv [48], focusing more on the $q^{2}$ dependence of the form factors, rather on their light quark mass dependence. Their predicted magnetic moments, with a $\left|g_{a}\right|=0.2$, are consistent with ours within uncertainties. 
[1] M. Mattson et al. (SELEX Collaboration), Phys. Rev. Lett. 89, 112001 (2002).

[2] A. Ocherashvili et al. (SELEX Collaboration), Phys. Lett. B 628, 18 (2005).

[3] F. S. Yu, H. Y. Jiang, R. H. Li, C. D. Lü, W. Wang, and Z. X. Zhao, Chin. Phys. C 42, 051001 (2018).

[4] W. Wang, F. S. Yu, and Z. X. Zhao, Eur. Phys. J. C 77, 781 (2017).

[5] W. Wang, Z. P. Xing, and J. Xu, Eur. Phys. J. C 77, 800 (2017).

[6] H. S. Li, L. Meng, Z. W. Liu, and S. L. Zhu, Phys. Lett. B 777, 169 (2018).

[7] L. Y. Xiao, K. L. Wang, Q. F. Lu, X. H. Zhong, and S. L. Zhu, Phys. Rev. D 96, 094005 (2017).

[8] E. L. Cui, H. X. Chen, W. Chen, X. Liu, and S. L. Zhu, Phys. Rev. D 97, 034018 (2018).

[9] D. B. Lichtenberg, Phys. Rev. D 15, 345 (1977).

[10] B. Julia-Diaz and D. O. Riska, Nucl. Phys. A739, 69 (2004).

[11] Y.s. Oh, D. P. Min, M. Rho, and N. N. Scoccola, Nucl. Phys. A534, 493 (1991).

[12] B. Patel, A. K. Rai, and P. C. Vinodkumar, arXiv: 0803.0221.

[13] N. Sharma, H. Dahiya, P. K. Chatley, and M. Gupta, Phys. Rev. D 81, 073001 (2010).

[14] A. Faessler, T. Gutsche, M. A. Ivanov, J. G. Korner, V. E. Lyubovitskij, D. Nicmorus, and K. Pumsa-ard, Phys. Rev. D 73, 094013 (2006).

[15] B. Silvestre-Brac, Few-Body Syst. 20, 1 (1996).

[16] S. K. Bose and L. P. Singh, Phys. Rev. D 22, 773 (1980).

[17] S. N. Jena and D. P. Rath, Phys. Rev. D 34, 196 (1986).

[18] H. S. Li, Z. W. Liu, X. L. Chen, W. Z. Deng, and S. L. Zhu, arXiv:1706.06458.

[19] U. Özdem, arXiv:1804.10921.

[20] K. U. Can, G. Erkol, B. Isildak, M. Oka, and T. T. Takahashi, J. High Energy Phys. 05 (2014) 125.

[21] X.-L. Ren, L. S. Geng, J. Martin Camalich, J. Meng, and H. Toki, J. High Energy Phys. 12 (2012) 073.

[22] Y. Xiao, X. L. Ren, J. X. Lu, L. S. Geng, and U. G. Meiner, Eur. Phys. J. C 78, 489 (2018).

[23] S. Weinberg, Physica A (Amsterdam) 96, 327 (1979).

[24] E. E. Jenkins and A. V. Manohar, Phys. Lett. B 255, 558 (1991).
[25] V. Bernard, N. Kaiser, and U. G. Meissner, Int. J. Mod. Phys. E 04, 193 (1995).

[26] T. Becher and H. Leutwyler, Eur. Phys. J. C 9, 643 (1999).

[27] T. Fuchs, J. Gegelia, G. Japaridze, and S. Scherer, Phys. Rev. D 68, 056005 (2003).

[28] L. Geng, Front. Phys. (Beijing) 8, 328 (2013).

[29] L. S. Geng, J. Martin Camalich, and M. J. Vicente Vacas, Phys. Rev. D 80, 034027 (2009).

[30] L. S. Geng, J. Martin Camalich, L. Alvarez-Ruso, and M. J. Vicente Vacas, Phys. Rev. Lett. 101, 222002 (2008).

[31] L. S. Geng, J. Martin Camalich, and M. J. Vicente Vacas, Phys. Lett. B 676, 63 (2009).

[32] X. L. Ren, L. S. Geng, and J. Meng, Phys. Rev. D 91, 051502 (2015).

[33] X. L. Ren, X. Z. Ling, and L. S. Geng, Phys. Lett. B 783, 7 (2018).

[34] X. L. Ren, L. S. Geng, and J. Meng, Phys. Rev. D 89, 054034 (2014).

[35] L. S. Geng, J. Martin Camalich, and M. J. Vicente Vacas, Phys. Rev. D 79, 094022 (2009).

[36] L. S. Geng, K. W. Li, and J. Martin Camalich, Phys. Rev. D 89, 113007 (2014).

[37] T. Ledwig, J. Martin Camalich, L. S. Geng, and M. J. Vicente Vacas, Phys. Rev. D 90, 054502 (2014).

[38] J. M. Alarcon, J. Martin Camalich, and J. A. Oller, Ann. Phys. (Amsterdam) 336, 413 (2013).

[39] Y. H. Chen, D. L. Yao, and H. Q. Zheng, Phys. Rev. D 87, 054019 (2013).

[40] V. Lensky and V. Pascalutsa, Eur. Phys. J. C 65, 195 (2010).

[41] A. N. Hiller Blin, T. Ledwig, and M. J. Vicente Vacas, Phys. Lett. B 747, 217 (2015).

[42] L. S. Geng, N. Kaiser, J. Martin-Camalich, and W. Weise, Phys. Rev. D 82, 054022 (2010).

[43] M. Altenbuchinger, L.-S. Geng, and W. Weise, Phys. Rev. D 89, 014026 (2014).

[44] D. L. Yao, M. L. Du, F. K. Guo, and U. G. Meiner, J. High Energy Phys. 11 (2015) 058.

[45] H. Xu, B. Wang, Z. W. Liu, and X. Liu, arXiv:1708.06918.

[46] Z. F. Sun and M. J. Vicente Vacas, Phys. Rev. D 93, 094002 (2016).

[47] D. L. Yao, Phys. Rev. D 97, 034012 (2018).

[48] A. N. H. Blin, Z. F. Sun, and M. J. Vicente Vacas, arXiv: 1807.01059. 